Gastroenterologe 2011 · 6:91

DOI 10.1007/s11377-010-0527-4

Online publiziert: 19. Februar 2011

(c) Springer-Verlag 2011
H.E. Blum ${ }^{1} \cdot$ M. Manns ${ }^{2}$

${ }^{1}$ Abteilung Innere Medizin II, Medizinische Universitätsklinik Freiburg

${ }^{2}$ Klinik für Gastroenterologie, Hepatologie und Endokrinologie, Medizinische Hochschule Hannover

\title{
Molekulare Diagnostik und Therapie bei gastrointestinalen Erkrankungen
}

Neben der Grundlagenforschung finden molekulargenetische Aspekte zunehmend Eingang auch in Klinik und Praxis. So erlauben molekulare Analysen ein zunehmendes Verständnis der molekularen $\mathrm{Pa}$ thogenese von gastrointestinalen Erkrankungen und ihrer Diagnose. Neben den im Beitrag von W. Stremmel und Mitarbeitern detailliert dargestellten hereditären Lebererkrankungen gibt es eine Vielzahl von gastrointestinalen Erkrankungen, wie z. B. die familiäre adenomatöse Polyposis (FAP) oder das hereditäre Kolonkarzinom (CRC), die sich im Einzelfall präsymptomatisch diagnostizieren lassen. Durch genomweite Assoziationsstudien (GWAS) können heute darüber hinaus „Single-Nucleotide-Polymorphismen“ (SNPs) nachgewiesen werden, die mit Persönlichkeitsmerkmalen (z. B. Körpergröße, Haarfarbe) bzw. mit individuellen Krankheitsanlagen assoziiert sind und dann für die Krankheitsprävention bzw. Früherkennung klinisch relevant sein können. Diese Einzelgenanalysen werden komplementiert durch die Array-Diagnostik, durch die Zehntausende von Genen (Genomics) bzw. deren Expressionsprodukte (Transkriptomics und Proteomics) sowie Metaboliten (Metabolomics) simultan analysiert werden können und eine krankheitsspezifische Signatur definieren lassen.

Die verschiedenen molekularen Analysen können jedoch nicht nur diagnostisch, sondern auch prognostisch und therapeutisch relevant sein. So lässt sich durch molekulare Analysen z. B. das Ansprechen einer Erkrankung auf eine bestimmte Therapie voraussagen. Ein aktu- elles klinisches Beispiel hierfür ist der Beitrag von I. Mederacke und T. von Hahn zur Hepatitis-C-Virus-Infektion. Weitere Beispiele sind das CRC und dessen Ansprechen auf Chemotherapeutika bzw. monoklonale Antikörper. So ist zu erwarten, dass durch molekulargenetische Analysen immer mehr Krankheits- bzw. Patientensubgruppen definiert werden können, die prognostisch und im Hinblick auf das optimale therapeutische Vorgehen unterschiedlich sind.

\section{(7) Aus der prinzipiellen Reversibiltät epigenetischer Modifikationen ergeben sich vielversprechende therapeutische Perspektiven}

Neben der Genetik und der molekulargenetischen Diagnostik ist die Epigenetik eines der aktuellsten Gebiete der biomedizinischen Forschung. Anders als die Genetik, die z.B. DNA-Mutationen und SNPs analysiert, befasst sich die Epigenetik mit kovalenten Modifikationen von DNA (Methylierung) und von Histonen (Acetylierung, Methylierung, Phosphorylierung, Ubiquitinylierung) sowie der Positionierung von Nukleosomen, die u. a. für die Regulation der Genexpression durch nichtkodierende Mikro-RNA-Spezies (miRNA) kritisch ist. So werden aberrante epigenetische Modifikationen zunehmend auch als krankheitsrelevant erkannt, beispielsweise für das hepatozelluläre Karzinom. Aus der prinzipiellen Reversibiltät epigenetischer Modifikationen ergeben sich vielversprechende therapeutische Perspektiven, vor allem für maligne Erkrankungen.
Perspektivisch ist $\mathrm{zu}$ erwarten, dass genetische und epigenetische Analysen in zunehmendem Maße nicht nur in die Diagnostik und Therapie von gastrointestinalen Erkrankungen, sondern z. B. durch GWAS auch in die präsymptomatische Abschätzung des individuellen Erkrankungsrisikos Eingang finden werden.

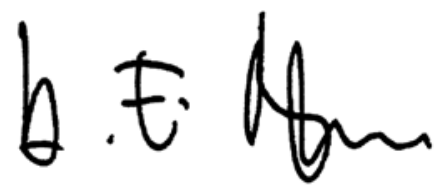

H.E. Blum

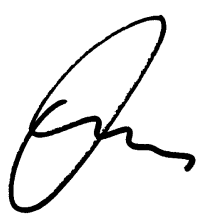

M.P. Manns

Korrespondenzadressen

Prof. Dr. Dr. h.c. mult. H.E. Blum

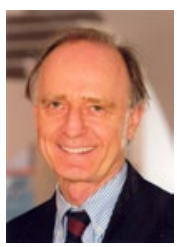

Abteilung Innere Medizin II,

Medizinische Universitäts-

klinik Freiburg

Hugstetter Strasse 55,

79106 Freiburg

hubert.blum@

uniklinik-freiburg.de

\section{Prof. Dr. M.P. Manns}

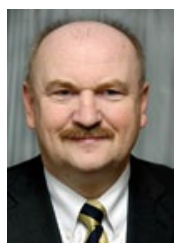

Klinik für Gastroenterologie, Hepatologie und Endokrinologie, Medizinische Hochschule Hannover Carl-Neuberg-Str. 1, 30623 Hannover Manns.michael@ mh-hannover.de 